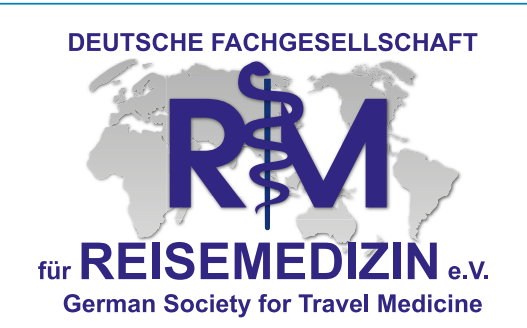

Hansaallee 299, 40549 Düsseldorf Tel.: 0211/5202581 Fax: 0211/5202583 E-Mail: info@fachgesellschaft-reisemedizin.de www.fachgesellschaft-reisemedizin.de

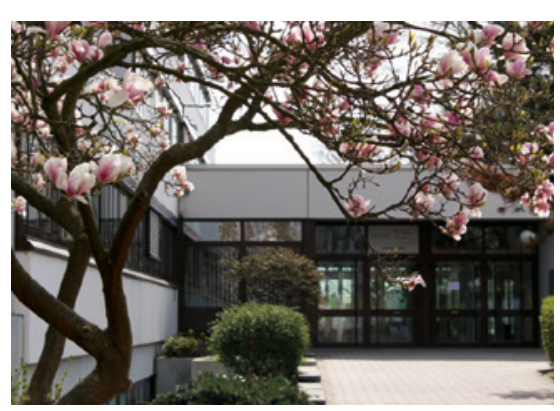

Hotel der Akademie Heinrich Pesch Haus Ludwigshafen am Rhein-Eingang. Quelle: Heinrich Pesch Haus, Ludwigshafen

\title{
Liebe Mitglieder der DFR,
}

vor wenigen Tagen ist Ihnen unser erstes Rundschreiben dieses Jahres zugegangen, zusammen mit der Beitragsrechnung für das Jahr 2016. Falls Sie unter die neue Beitragsregelung für Ruheständler fallen, wenden Sie sich bitte an die Geschäftsstelle. Frau Bergmann bedankt sich übrigens für die vielen Besuche von Mitgliedern und guten Gespräche an unserem Stand beim letzten Forum ,Reisen und Gesundheit' in Berlin. Dabei konnten einige neue Mitglieder gewonnen werden, auch unser Qualitätshandbuch wurde stark nachgefragt.

Da die Anerkennung der DFR als gemeinnütziger Verein vom zuständigen Finanzamt bereits schriftlich in Aussicht gestellt wurde, können die nächsten Schritte zur Stärkung der Fachgesellschaft vorbereitet werden. Bitte beachten Sie dazu die Hinweise im Rundschreiben und die erbetenen Rückmeldungen.

Das Programm für unsere Jahrestagung in Ludwigshafen ist weitgehend fertiggestellt; es beweist wieder einmal die Breite und Tiefe der Reisemedizin: im umfassenden Sinne die Reisenden ,fit for travel ${ }^{\star}$ zu machen.

Zuvor, nämlich am 30. April 2016 in Reutlingen, ist unser Arbeitskreis ,Reisemedizin und Öffentlicher Gesundheitsdienst' mit einem Satellitensymposium bei der wissenschaftlichen Jahrestagung des Bundesverbands der Ärzte im ÖGD (BVÖGD) vertreten - nach Magdeburg und Rostock bereits zum dritten Mal, auf ausdrücklichen Wunsch der Teilnehmer. Falls Sie am Samstag, 30. April, in der Nähe von Reutlingen sind und gerne teilnehmen möchten, würde ich mich sehr freuen. Das Programm und den Link zur Anmeldung finden Sie auf unserer Homepage: www. fachgesellschaft-reisemedizin.de.

Wir haben bereits mehrfach auf die 6. Northern European Conference on Travel Medicine (NECTM), die vom 1. bis 4 . Juni 2016 in London stattfindet, hingewiesen. Die DFR ist Mitveranstalter und mit mehreren Postern und Vorträgen vertreten. Der Vorstand würde sich sehr freuen, wenn Sie in London zusammen mit uns die Fachgesellschaft vertreten. Am Ende noch eine persönliche Bemerkung von mir zum Tode unserer hochgeschätzten Kollegin Dr. Ursula Mikulicz. Für meine Vorstandstätigkeit stand sie mir jederzeit mit gutem Rat zur Seite - in unruhigen Zeiten war sie ein Ruhepunkt - das Wohl der DFR verlor sie nie aus dem Fokus. Ihr klares, manchmal pointiertes Auftreten bei den Mitgliederversammlungen, war oft zielführend und ,Schwert für einen gordischen Knoten'. Herrn Kollegen Burkhard Rieke herzlichen Dank für seinen Nachruf!

\section{Mit herzlichen Grüßen}

Ihr

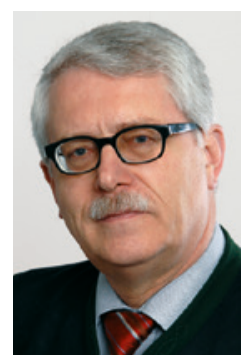

Günter Schmolz, Bietigheim-Bissingen 


\section{Zum Tode von Dr. Ursula Mikulicz}

Völlig überraschend und aus voller Aktivität heraus ist am 10. Februar 2016 in Kronberg unser langjähriges Vorstandsmitglied und zeitweise Stellvertretende Vorsitzende Frau Dr. Ursula Mikulicz im Alter von 81 Jahren verstorben. Vielen innerund außerhalb der Reiseme-

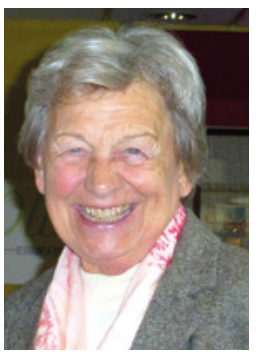

Jahren. Aus dieser Zeit stammt auch ihr Einsatz in Gremien der Berufsgenossenschaften, sei es rund um die Vorschriften zur G35, der „Tropentauglichkeitsuntersuchung“ oder als Gutachterin in komplizierten Fällen. Der HIV-Pandemie und den posttraumatischen Belastungsstörungen galt im dizin wird sie als engagierte, ja kämpferische Verfechterin eines länder- und kulturenübergreifenden Verständnisses der Medizin in bester Erinnerung bleiben.

Ursula Mikulicz, die mit 10 Jahren dem untergehenden Berlin nur knapp entkommen war, studierte Medizin in Göttingen - die DFR-Jahrestagung dort 2009 war ein „Heimspiel“ für sie - und durchlief die Weiterbildung zur Kinderärztin an der Bremer Rotkreuzklinik. 1964 bis 1968 ging sie in ein erstes Projekt nach Marokko, anschließend mit der GTZ nach Abidjan/Côte d'Ivoire - Stationen, die ihren Blick für soziale Benachteiligung schärften und ihr Verständnis der Medizin als konkreter Form des Handelns zugunsten des Mitmenschen definierten. Ab 1980 übernahm sie bis zu ihrem Ruhestand die Tropenmedizinische Untersuchungsstelle der GTZ (heute GIZ) in Eschborn und machte sie mit fachlichem Können, großer Auslandserfahrung, viel politischem Geschick und, wenn nötig, Kampfgeist, zu „einer Institution“ in der deutschen Entwicklungszusammenarbeit. Noch heute treffe ich immer wieder langjährige Experten in internationalen Diensten, die von dem Gefühl des „Aufgehobenseins“ berichten, das Ursula Mikulicz durch ihr Engagement für die Erkrankten und Verunglückten bewirkte. In endlosen Telefonaten bewegte sie Luftlinien, Rettungsdienste, Versicherer und Kliniken so manches Mal, über den eigenen Schatten zu springen. Meine ersten Telefonate mit ihr waren solche im tropenmedizinischen Nachtdienst in den 90er Hinblick auf die Fachkräfte ihre besondere Aufmerksamkeit. Mit der Wahl in den Vorstand unserer Fachgesellschaft stellte sie ihren Erfahrungsschatz und ihre zahlreichen Kontakte in den Dienst einer Medizin, die Grenzen überwinden will. Vielfältige Anregungen kamen von ihr, sei es im Kontakt mit der Bundesärztekammer, auf internationaler Ebene, rund um die Exkursionen oder für die Gestaltung der Jahrestagungen. Daneben entfaltete sie gerade im Ruhestand ein vielfältiges Engagement: Beispielhaft genannt seien die Alumni der Studienstiftung, für die sie etwa die Tagung in der Frankfurter Universität im November 2013 zusammenstellte, aber auch die individuelle Förderung, mit der sie die Schüler einer benachbarten Gesamtschule in Praktika und Lehren brachte durch Hilfe bei Bewerbungsschreiben, Training für Interviews und durchaus konstruktive Kritik. Vielen von ihnen vermittelte sie ein völlig neues Selbstbild, indem sie ihnen die Möglichkeit vor Augen führte, Ziele und Erfolg zu haben, wenn sie sich nur für eine Hartz-IVKarriere berufen fühlten.

Mit Frau Dr. Mikulicz ist eine aufrechte, überaus engagierte und erfahrene Kollegin viel zu früh von uns gegangen, die den klinischen Blick zum Blick für Benachteiligung weitete und die Medizin als Auftrag zum Brückenschlag verstand, über Sprach- und Kulturgrenzen hinweg. Wir werden ihr ein ehrendes Andenken bewahren.

Im Auftrag des Vorstands der Deutschen Fachgesellschaft für Reisemedizin Burkhard Rieke, Düsseldorf 


\section{Jahrestagung der Deutschen Fachgesellschaft für Reisemedizin e. V.}

\section{3.-24. September 2016 in Ludwigshafen}

Veranstaltungsort: Heinrich Pesch Haus • Frankenthaler Straße 229 • 67059 Ludwigshafen

Freitag, 23. September

$\begin{aligned} \text { 09:00-11:30 Uhr } & \text { Workshop für Studierende } \\ & \text { Einführung in die Reisemedizin mit Kasuistiken } \\ & \text { Dr. Ulrich Klinsing }\end{aligned}$
ab 12:30 Uhr
13:00-13:15 Uhr Begrüßung
Prof. Dr. Günter Schmolz

$\begin{array}{ll}\text { 13:15-13:55 Uhr } \quad \text { Kasuistiken } & \\ & \text { Dr. Rosemarie Mazzola }\end{array}$

13:55-14:40 Uhr Globalisierung 3.0:

weltweite Schicksalsgemeinschaft

Prof. Dr. Dr. Johannes Wallacher

14:40-15:00 Uhr Kaffeepause

15:00-15:45 Uhr Gesundheitssysteme, gesundheitliche

Versorgung im Ausland

Dr. Stefan Eßer

15:50-17:15 Uhr FSJ Blickwechsel mit anschließender Diskussion Christian Weinert

\section{7:15 Uhr \\ 18:30 Uhr}

Mitgliederversammlung

Transfer ab Heinrich Pesch Haus

Führung durch die Weinkellerei der BASF

Abendessen im Gesellschaftshaus der BASF

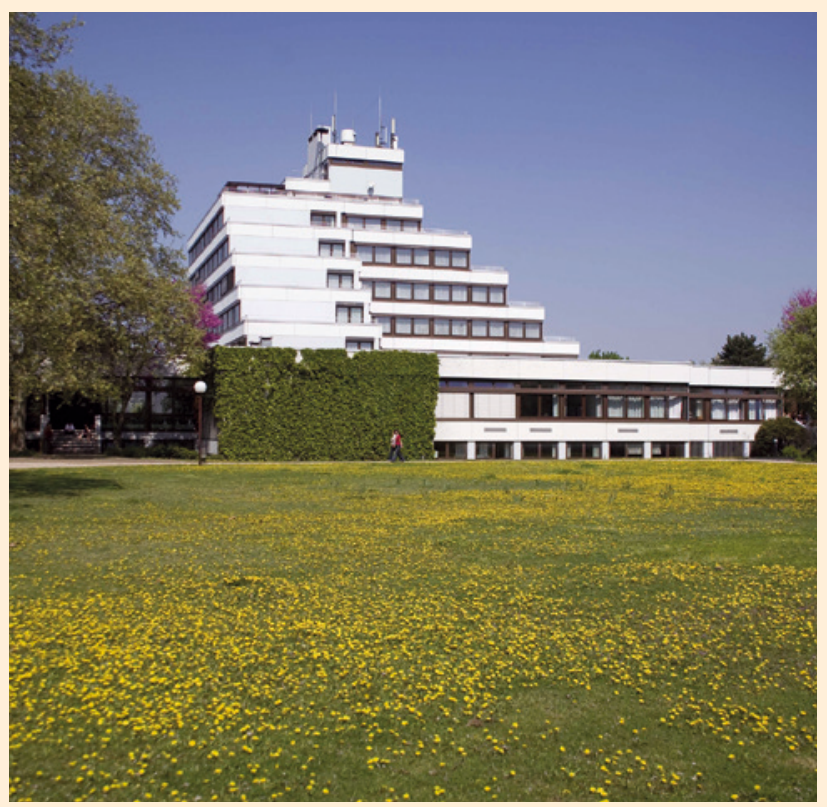

Hotel der Akademie Heinrich Pesch Haus Ludwigshafen

Quelle: Heinrich Pesch Haus, Ludwigshafen
Samstag, 24. September

09:00-09:45 Uhr Weltseuchenlage, Update Malaria,

Update Impfungen

Dr. Burkhard Rieke

09:45-10:30 Uhr

Beratung und Betreuung von Expats einschließlich der Familie und bei Vorerkrankungen N.N., Arbeitsmedizinische Abteilung, BASF

\section{0:30-10:50 Uhr Kaffeepause}

10:50-11:20 Uhr Ambulante Traumatologie in der Reisemedizin Dr. Wolfgang Lorenz

11:20-11:50 Uhr Traumatologie stationär bei Rückkehrern Prof. Dr. Paul Alfred Grützner

11:50-12:20 Uhr Zahnmedizin unterwegs Dr. Sabrina Strobel

12:20-13:00 Uhr

13:00-13:30 Uhr

13:30-14:15 Uhr Mittagspause

Drogenkonsum, Drogenbesitz und rechtliche Konsequenzen bei Reisenden - länderspezifisch Dr. Ingo Ilja Michels

Substitutionspatienten auf Reisen Ralf Gerlach

14:15-14:45 Uhr Koadministration von Impfungen insbesondere bei Last-Minute-Reisen Prof. Dr. Ulrich Heininger

14:45-15:10 Uhr Kaffeepause

15:10-15:50 Uhr Import von multiresistenten Erregern bei Reiserückkehrern - Konsequenzen für die Reisemedizin Dr. Sophie Schneitler

15:50-16:35 Uhr Überblick über übertragbare und nicht übertragbare Erkrankungen von Asylsuchenden Dr. Rainer Schwertz

16:35-17:05 Uhr Nachwuchsforscher stellen sich vor, evtl. mit Preisverleihung

17:05 Uhr Ausblick und Verabschiedung Prof. Dr. Günter Schmolz

Die Zertifizierung der Veranstaltung wird bei der zuständigen Landesärztekammer beantragt.

Informationen und Anmeldung

Deutsche Fachgesellschaft für Reisemedizin e.V.

Hansaallee 299 - 40549 Düsseldorf

Tel.: 02115202581 - Fax: 02115202583

E-Mail: gs@fachgesellschaft-reisemedizin.de www.fachgesellschaft-reisemedizin.de 